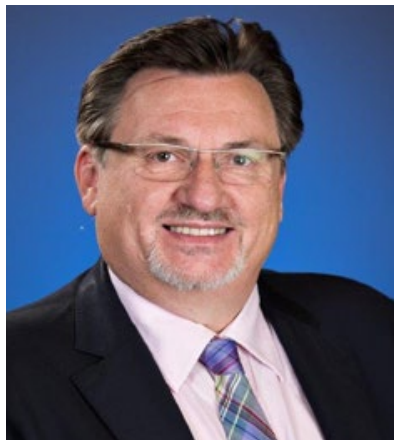

Franz Wagner

Geschäftsführer des DBfK
Deutscher Pflegerat e.V.

Bundesarbeitsgemeinschaft

Pflege- und Hebammenwesen

In Kooperation mit

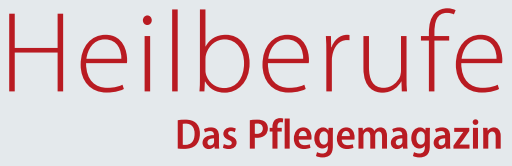

\title{
Editorial
}

\section{Aktion: Mein Recht auf Frei!}

V ertrauen und Verlässlichkeit sind zwei sehr hohe Werte, die es zu schützen gilt. Das sollte eine Selbstverständlichkeit sein - ist es aber nicht immer. Ganz besonders gilt dies, wenn es um die Dienstplanung und die Festlegung geht, wie viel Personal denn tatsächlich für die Leistungserbringung nötig ist. Zu den Auswirkungen der seit Jahren kontinuierlich unzureichenden Pflegepersonalbemessung in Pflegeeinrichtungen und Krankenhäusern gehören nicht nur hohe und weiter steigende Krankheitsausfälle. Problematisch sind für Patienten und Bewohner, wie auch für die Beschäftigten in der Pflege, die Lücken und die Unterbesetzung, die bei kurzfristigem Personalausfall und/oder Arbeitsspitzen entstehen.

In einer bundesweiten Aktion weist der DBfK jetzt darauf hin, dass das gesicherte, respektierte Dienstfrei und die Verlässlichkeit des Dienstplans ein hohes Gut der Wertschätzung der Mitarbeiterinnen und Mitarbeiter sind. Gleiches gilt für das Einhalten von rechtskonformen Arbeitspausen.

Von der Bundespolitik hören wir immer wieder, dass die Umsetzung des neuen Pflegebedürftigkeitsbegriffes mit den aktuellen Personalschlüsseln nicht möglich sei. Hierfür werde mehr Personal benötigt. Dem stimmen wir als Deutscher Pflegerat voll und ganz zu. Dafür müssen sich aber die Einrichtungen, deren Verbände und die Kostenträger der Kassen und Sozialhilfeträger schnellstmöglich bewegen und mit der Vereinbarung für mehr Personal bessere Rahmenbedingungen für die Pflege schaffen.

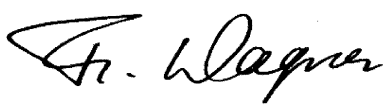

Franz Wagner

Vize-Präsident des Deutschen Pflegerats (DPR)

\section{IM FOCUS}

\section{Gemeinsam die Zukunft der Pflege gestalten}

Der Deutsche Pflegetag 2016 findet vom10. bis 12. März 2016 in Berlin statt. Bundesgesundheitsminister Hermann Gröhe hat die offizielle Schirmherrschaft für den Deutschen Pflegetag 2016 übernommen und wird zurm Thema "Herausforderung Pflege" sprechen. Das ist ein starkes Zeichen für die professionell PflegendePflegenden.

Zwei Kongresstage mit vielen spannenden Veranstaltungen schließen sich an. Am Freitag werde ich zu Beginn des Kongresstages unsere Wünsche und Forderungen sowie Lösungsmöglichkeiten darlegen. Danach geht es in die Programmblöcke „Wie Pflegende Politik machen können", die „Zukunft der stationären Pflege" und „Psychische Belastungen und Ressourcen des Pflegeberufs", um nur einige zu nennen.

Das „Cockpit Pflege" mit pflegepolitischen Sprecher/innen der Fraktionen im Deutschen Bundestag sowie die Erstveröffentlichung des Pflegethermometer 2016 schließen sich an, bevor es dann ausführlich um die uns betreffenden neuen Gesetze geht. Dem Pflegeberufsgesetz wird dabei ein eigener Veranstaltungsblock gewidmet.

Am Samstag stehen u. a. die Themen „Personalbemessung“, ,Pflegebildung" und die „Pflegewirtschaft im Wandel“ im Mittelpunkt. Der Deutsche Pflegetag wird sich darüber hinaus den Themen Reha und Hygiene genauso widmen, wie dem Zusammenwirken von pflegenden Angehörigen und professionellen Helfern.

Die Fragen, welche Herausforderung die Gesundheitsversorgung von Flüchtlingen an uns stellt und wie der Stand zum Thema Pflegekammer ist, runden das Programm ab.

Andreas Westerfellhaus

Präsident des Deutschen Pflegerats 